\title{
Research on Inter-harmonic Detection Method Based on EEMD
}

\author{
Sun $\mathrm{Jie}^{1}$, Tao Shuyi ${ }^{1}$, Han Chao ${ }^{2}$, Li Gang $^{1}$, Lan Chenghao ${ }^{1}$, Zhao Qidong $^{1}$, \\ Song Yuanlin ${ }^{1}$, Sun Jiatong ${ }^{1}$ \\ ${ }^{1}$ Fushun Power Supply Company, Liaoning Electric Power Company Limited, State Grid, China \\ ${ }^{2}$ Shenyang Power Supply Company, Liaoning Electric Power Company Limited, State Grid, China
}

Keywords: empirical mode decomposition; ensemble empirical mode decomposition; Hilbert transform; inter-harmonic; detection

\begin{abstract}
A comparative analysis of the existing inter-harmonic detection methods are carried out for the small content, non-linear and volatility of characteristic. Based on HHT transform theory, a new inter-harmonic detection method based EEMD is presented by combining of the empirical mode decomposition and the ensemble empirical mode decomposition algorithm. The signal is decomposed into a single-component signal by EEMD, and then the frequency and amplitude of each component is accurately calculate through HHT transform, and the accuracy of this method is verified through simulation and measurement. Compared with the EMD algorithm, the proposed method can eliminate the false components and mode mixing and be suitable for the detection of inter-harmonic signal.
\end{abstract}

\section{Introduction}

With the nonlinear element of power electronic devices are widely used in the power grid, the grid, in addition to produce integer multiples of the fundamental frequency of harmonic frequencies, but also the fundamental frequency harmonics may arise between non-integer frequencies. Even low harmonic amplitude, in addition to such as electrical equipment, such as overheating and reducing the life of the typical problems being caused, some new problems are brought to power, summarized as follows synchronous oscillations, voltage fluctuations and flicker [1-3].

From the time domain or frequency-domain to time frequency domain combination detection method, approach based on FFT and DFT is the major means of harmonic detection. However, only a global Fourier transform is exist, or completely in the time domain, or completely in the frequency domain , so it requires the system to be linear, and the data must be strictly periodic or stationary [4].This determines limitations about the inter-harmonic detection methods. Based on this, harmonic and inter-harmonic detection methods based on the empirical mode decomposition (Empirical Mode Decomposition, EMD) are proposed in the literature [5] and [6]. This method draws on the advantages of multi-resolution wavelet transform, while difficulty of the need to select the wavelet transform wavelets is overcome, from the signal scale features starting signal decomposition, and the characteristics of the signal can be analyzed as soon as possible, with good local adaptation [7]. However, some shortcomings exist in the EMD decomposition process, which can affect inter-harmonics test results. The more serious problem is prone to false component and modal aliasing [8], in particular in: 1) containing a separate IMF disparate scales; 2) the same scale in various IMF.

For some of the shortcomings of EMD, a new method is proposed by this article for harmonic detection based on the overall average empirical mode decomposition (Ensemble Empirical Mode Decomposition, EEMD).EEMD and Hilbert-Huang transform (Hilbert-Huang Transform, HHT) are combined by this method, first signal EEMD decomposed to get intrinsic mode function, and then making Hilbert transform, so inter-harmonic signal amplitude value and frequency are found. The accuracy and validity of the proposed method is verified through simulation. 


\section{EEMD and HHT principles}

\section{EMD Principle}

Signal can be decomposed into a set of empirical mode function by EMD method, and specifically the decomposition process is as follows [9].

First calculated upper envelope $v_{1}(t)$ and lower envelope $v_{2}(t)$ according to the local maximum points and minimum points on the signal ${ }^{x(t)}$, and then calculated the mean envelope to the mean curve $m_{1}(t)=\frac{1}{2}\left(v_{1}(t)+v_{2}(t)\right)$, and then find the difference recorded as $h_{1}(t)$ between $x(t)$ and $m_{1}(t)$ that

$$
x_{1}(t)-m_{1}(t)=h_{1}(t)
$$

$h_{1}(t)$ is treated as new $x(t)$ to repeat the above operation, get

$$
h_{1}(t)-m_{1.1}(t)=h_{1.1}(t)
$$

$k$ is second screening continues until $h_{1, k}(t)$ IMF condition is met, remember

$$
c_{1}(t)=h_{1, k}(t)
$$

$c_{1}(t)$ is considered as the first IMF.

Find the original signal and $x(t)$ difference of $c_{1}(t)$

$$
x_{1}(t)-c_{1}(t)=r_{1}(t)
$$

$r_{1}(t)$ is considered new ${ }^{x(t)}$, the above procedure is repeated sequentially to obtain a second IMF, $c_{2}(t)$, third IMF, ${ }^{c_{3}(t)}$ to $n_{\mathrm{IMF}} c_{n}(t)$, until $r_{n}(t)$ substantially monotonic trend or $\left|r_{n}(t)\right|$ is small that can be considered as the measurement error when to stop. So by the above decomposition process the original signal is decomposed into $\mathrm{n}$ IMF and a residual component and

$$
x(t)=\sum_{i=1}^{n} c_{i}(t)+r_{n}(t)
$$

In order to ensure the IMF screened out enough physical significance in the amplitude and frequency, the size of $i$ standard deviation of two consecutive processing results before and after as the criterion of intrinsic mode functions:

$$
S D=\sum_{t=0}^{T} \frac{\left[h_{k-1}(t)-h_{k}(t)\right]^{2}}{h_{k-1}^{2}(t)}
$$

$S D$ value is ranged from 0.2 to 0.3 .

\section{EEMD Principle}

In order to eliminate spurious components and modal aliasing in EMD, EEMD algorithm is proposed [10]. The white noise is added to the desired signal decomposition to add some missing scales, the use of white noise with a uniform distribution of the statistical characteristics of the frequency mode to eliminate aliasing effects, the algorithm steps are as follows.

(1) Adding $\omega(t)$ group to the original white noise signal $x(t)$ to obtain in the overall signal $^{X(t)}$;

$$
X(t)=x(t)+\omega(t)
$$

(2) ${ }^{X(t)}$ carried on EMD decomposition, each IMF component;

$$
X(t)=\sum_{j=1}^{n} c_{j}(t)+r_{n}
$$

(3) The original signal ${ }^{x(t)}$ adding different white noise $\omega_{i}(t)$, repeat the above steps, the 
decomposition of each IMF component group;

$$
X_{i}(t)=\sum_{j=1}^{n} c_{i j}(t)+r_{i n}
$$

(4) The corresponding mean of the IMF as the final decomposition result.

$$
c_{n}(t)=\frac{1}{N} \sum_{i=1}^{n} c_{i, n}(t)
$$

Frequency of Adding white noise obeys statistical regularities:

$$
\varepsilon_{n}=\frac{\varepsilon}{\sqrt{N}}
$$

Where, $N$ is a whole number; $\varepsilon^{\varepsilon}$ adding white noise amplitude; ${ }^{\varepsilon_{n}}$ is the error signal to reconstruct the original signal with a resolution IMF was obtained. By the formula (11) shows that the amplitude of the noise is constant, the larger $N$ the closer the final decomposition true value; the noise amplitude values should be appropriate.

\section{HHT Principle}

For either a continuous-time signal ${ }^{x(t)}, \hat{x}(t)$ which Hilbert transform is defined as

$$
\hat{x}(t)=\frac{1}{\pi} \int_{-\infty}^{+\infty} \frac{x(\tau)}{t-\tau} d \tau
$$

Its inverse transform is:

$$
x(t)=\frac{1}{\pi} \int_{-\infty}^{+\infty} \frac{\hat{x}(\tau)}{\tau-t} d \tau
$$

The ${ }^{x(t)}$ and ${ }^{\hat{x}(t)}$ consisting of analytic signal

$$
Z(t)=x(t)+i \hat{x}(t)=a(t) e^{i \phi(t)}
$$

Instantaneous amplitude of the signal $a(t)$

$$
a(t)=\left[x(t)^{2}+\hat{x}(t)^{2}\right]^{\frac{1}{2}}
$$

The instantaneous phase $\cos \phi(t)$ is

$$
\phi(t)=\arctan \frac{\hat{x}(t)}{x(t)}
$$

Instantaneous frequency $f(t)$ is

$$
f(t)=\frac{1}{2 \pi} \frac{d \phi(t)}{d t}
$$

\section{Inter-harmonic detection and analysis}

EEMD signal is obtained containing a series of inter-harmonic of the IMF, IMF each stationary single-frequency signal, by means the Hilbert transform further to get the signal spectrum on the basis , therefore, the real physical processes is reflected by results based on the component of the Hilbert IMF special transformed in order to achieve inter-harmonic detection. The specific steps are:

(1) Using EEMD to decompose the original signal containing harmonics decomposition, get a group of IMF;

(2) For an IMF component of the Hilbert transform to obtain analytical expressions;

(3) Using formula (15) to (17) to obtain the instantaneous amplitude and instantaneous frequency of each mode component.

\section{Simulation}

To verify EEMD application effect in the harmonic detection, to simulate the signal, the 
inter-harmonic detection between EEMD and EMD harmonic detection results were compared to verify the accuracy of the algorithm.

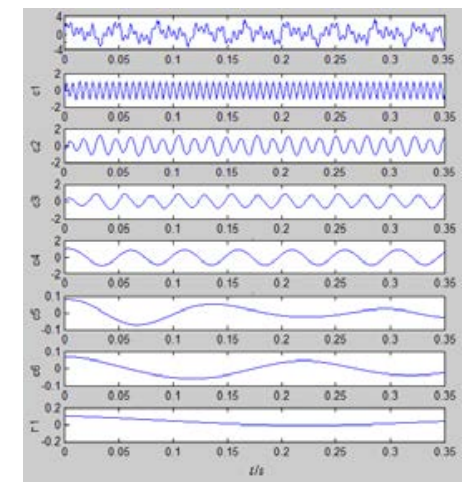

Fig. 1 The original signal and results by EMD

Let the simulated signal is

$u(t)=\sin 50 \pi t+\sin 100 \pi t+\sin 170 \pi t+\sin 400 \pi t$

Signal contains the fundamental signal and frequency of $25 \mathrm{~Hz}, 85 \mathrm{~Hz}$ inter-harmonics and harmonics of the $200 \mathrm{~Hz}$ signal. By EMD signal the decomposition results are shown in Figure 1. Figure 1 shows that, after the original signal is decomposed by EMD, given 6 single-frequency components of $c_{1}$ to $c_{6}$ IMF and the remaining components $r$.

Hilbert transform to each component, the amplitude and frequency of each component of the IMF obtained are shown in Figure 2.

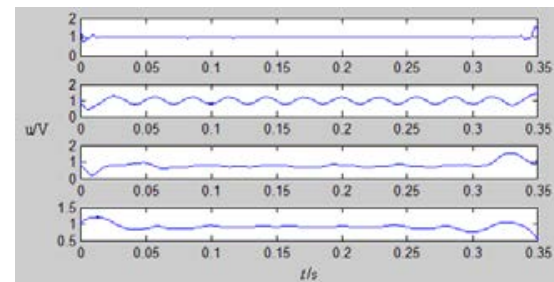

(a) Amplitude

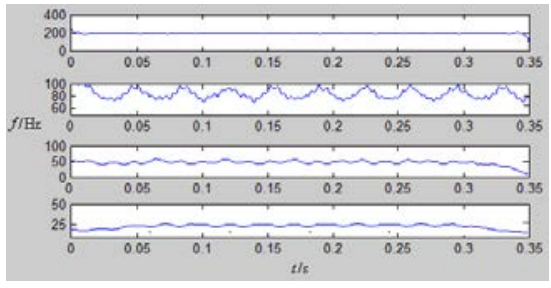

(b) Frequency

Fig. 2 The amplitudes and frequencies of IMFs by EMD

The amplitude and frequency of each component of the IMF obtained by HHT and EEMD algorithm for the same signal are shown in Figure 3 and 4.

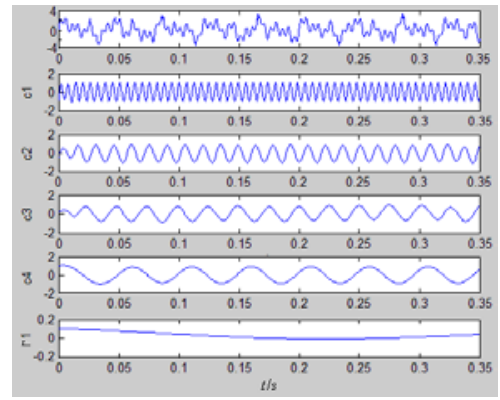

Fig. 3 The original signal and results by EEMD

Further, in Figure 1 obtained the second of IMF by EMD component appears obvious aliasing mode, comparison, Figure 3 by EEMD decomposition the second components of IMF clearly a sinusoidal component, the elimination modal aliasing.

By comparing Figures 2 and 4 amplitude and frequency IMF component obtained by the two algorithms, apparently the use of inter-harmonic detection EEMD algorithm, the accuracy is better than EMD algorithm. 


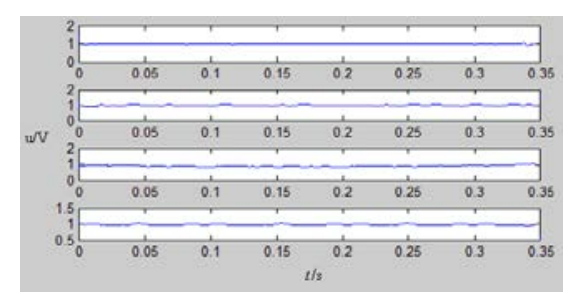

(a) Amplitude

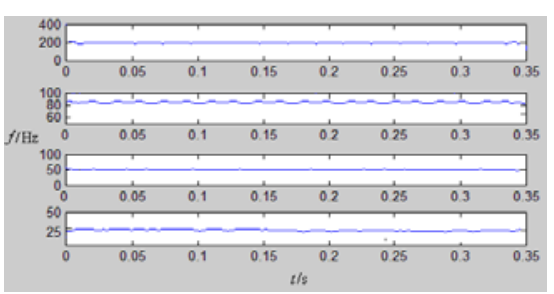

(b) Frequency

Fig. 4 The amplitudes and frequencies of IMFs by EEMD

\section{Conclusion}

The new method is proposed for inter-harmonic detection combining EMD and EEMD algorithm, then using HHT algorithm to detect inter-harmonics. Simulation results show that: Compared with the EMD algorithm, by EEMD algorithm for inter-harmonic detection, false and modal component aliasing appearing in the former decomposition process can be eliminated, and the result is more accurate; Experimental results show that the algorithm is accurate and feasible.

\section{References}

[1] A. Testa, M. F. Akram, R. Burch. Interharmonics: Theory and Modeling. IEEE Transactions on Power Delivery, Vol. 22, April, 2007, pp. 2335-2348.

[2] GB/T 24337-2009 Power quality Public power system interharmonics. Beijing: Standard of China, 2009.

[3] HAO Jiangtao, Liu Nian, Xing Jinyu, at el. The Sources and harms of interharmnics in power system. Sichuan Power Technologies, February, 2005, pp. 11-14.

[4] Zhang Xianda, Bao Zheng. Non-stationary signal analysis and processing. Beijing: National Defense Industry Press, 1998.

[5] LI Tianyun, ZHAO Yan, Li Nan, et al. A New Method for Power Quality Detection Based on HHT. Proceedings of the CSEE, Vol.25, August, 2005, pp. 52-56.

[6] WANG Hailu. Application of Empirical Mode Decomposition and Hilbert Transform on Detection of Harmonics and Inter-Harmonics. Telecom Power Technology, Vol.27, January, 2010, pp. 43-46.

[7] LI Tianyun, ZHAO Yan, Li Nan. Apply Empirical M ode Decomposition Based Hilbert Transform to Power System Transient Signal Analysis. Automation of Electric Power systems, Vol. 29, April, 2005, pp. 49-52.

[8] ZHU Ninghui, BAI Xiaomin, DONG Wejie. Harmonic Detection Method Based on EEMD. Proceedings of the CSEE, Vol. 33, July, 2013, pp. 93-98.

[9] N. E. Huang, Z. Shen, S. R. Long. The empirical mode decomposition and the Hilbert spectrum for nonlinear and non-stationary time series analysis. Proc R Soc London, A 454, 1998, pp. 903-995. 